\title{
Asymmetric Synthesis of 3-Hydroxy-pyrrolidines via Tin- Lithium Exchange and Cyclization
}

\author{
Guido Christoph ${ }^{\dagger}{ }^{\dagger}$ Christian Stratmann, ${ }^{\dagger}$ Iain Coldham ${ }^{*, \star}$ and Dieter Hoppe ${ }^{*, \dagger}$ \\ Supporting Information \\ Organisch-Chemisches Institut, Westfälische Wilhelms-Universität Münster, \\ Corrensstraße 40, D-48149 Münster, Germany \\ Department of Chemistry, University of Sheffield, Sheffield S3 7HF, UK
}

General Information. All ${ }^{1} \mathrm{H}$ and ${ }^{13} \mathrm{C}$ NMR spectra were recorded using Varian Inova 500 or Bruker ARX300, AMX400 and DRX400 spectrometer at ambient temperature in $\mathrm{CDCl}_{3}$. Chemical shifts are reported in parts per million as follows: chemical shift, multiplicity ( $\mathrm{s}=$ singlet, $\mathrm{d}=$ doublet, $\mathrm{t}=$ triplet, $\mathrm{q}=$ quartet, $\mathrm{m}=$ multiplet), coupling constant, integration and assignment. Infrared spectra were recorded on a Nicolet FT-IR 5DXC or FT-IR Magna 550 spectrophotometer. Mass spectra were obtained on a Micromass Quattro LC-Z or on a Finnegan MAT8200 spectrometer. Optical rotations were recorded on a Perkin-Elmer 341 or Optical Activity AA-1000 polarimeter at $589 \mathrm{~nm}$, and were reported as $[\alpha]^{23}$ (concentration in grams $/ 100 \mathrm{~mL}$ solvent). Analytical thin layer chromatography was performed using Merck $60-\mathrm{F}$ 254 plates. Flash column chromatography was achieved on Merck silica gel 43-60 $\mu \mathrm{m}$. Chiral HPLC analysis was excuted using an Waters 717 HPLC with photo diode array detector and a Chira-Grom 2 (Grom Analytik GmbH, 250*2 mm) column. All reactions were performed under inert atmosphere, in oven-dried glassware with magnetic stirring. Diethylether was dried over sodium and distilled before using. TMEDA was refluxed over $\mathrm{CaH}_{2}$ for $10 \mathrm{~h}$ and distilled. All other reagents were used as delivered from supplier.

\section{2-(Benzylamino)ethyl 2,2,4,4-tetramethyl-1,3-oxazolidine-3-carboxylate (4)}

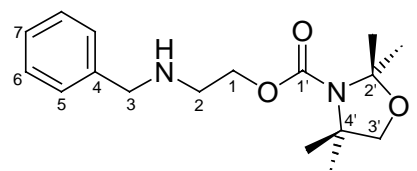

$2.44 \mathrm{~g}$ (61.0 mmol, 1.3 equiv) $\mathrm{NaH}(60 \%$ in mineral oil) were suspended in $100 \mathrm{~mL}$ THF and a solution of $7.00 \mathrm{~g}$ (46.9 mmol) 2-(benzylideneamino)-ethanol (2) in $50 \mathrm{~mL}$ THF was slowly added via a dropping funnel. The mixture was stirred for $1 \mathrm{~h}$ at room temperature and $10.8 \mathrm{~g}$ (56.3 mmol, 1.2 equiv) $\mathrm{CbyCl}$ were added. The reaction mixture was heated to reflux for $7 \mathrm{~h}$ and stirred at room temperature over night. After aqueous work up and drying over $\mathrm{Na}_{2} \mathrm{SO}_{4}$ the carbamate was obtained as yellow oil. This crude product $(17.2 \mathrm{~g})$ was dissolved in $100 \mathrm{~mL}$ $\mathrm{MeOH}$ (p. A.) and $5.28 \mathrm{~g}$ (141 mmol, 3.0 equiv) $\mathrm{NaBH}_{4}$ were added in small portions at $0{ }^{\circ} \mathrm{C}$. 
The mixture was stirred at room temperature for $3 \mathrm{~h}$ and then quenched with $2 \mathrm{~N} \mathrm{HCl} \mathrm{until} \mathrm{pH} 2$ was reached. Subsequently the solution was made alkaline with aq. $\mathrm{NaOH}$ and extracted three times with $50 \mathrm{~mL} \mathrm{Et}{ }_{2} \mathrm{O}$. After drying the organic layer with $\mathrm{MgSO}_{4}$ the solvent was removed in vacuum. Flash chromatography $\left(\mathrm{Et}_{2} \mathrm{O} / \mathrm{PE}=1: 1 \rightarrow \mathrm{Et}_{2} \mathrm{O}\right)$ of the crude product on desactivated silica gave 4 (10.2 g, $33.2 \mathrm{mmol}, 71 \%$ over 2 steps) as colourless oil.

${ }^{1} \mathrm{H}-\mathrm{NMR}\left(400 \mathrm{MHz}, \mathrm{CDCl}_{3}\right): \delta / \mathrm{ppm}=1.35,1.42,1.51,1.56\left(4 \times \mathrm{s}, 12 \mathrm{H} \mathrm{CH}_{3}(C b y)\right) ; 2.91(\mathrm{dt}, 2 \mathrm{H}$, $\left.{ }^{3} J_{1,2}={ }^{2} J_{2 \mathrm{a}, 2 \mathrm{~b}}=5.3 \mathrm{~Hz}, \quad \mathrm{H}-2\right) ; 3.72 \quad\left(\mathrm{~s}, 2 \mathrm{H}, \quad \mathrm{H}-3{ }^{\prime}\right) ; 3.83 \quad(\mathrm{~s}, 2 \mathrm{H}, \quad \mathrm{H}-3) ; 4.22$ (dt, 2H, $\left.{ }^{3} J_{1,2}={ }^{2} J_{1 \mathrm{a}, 1 \mathrm{~b}}=5.3 \mathrm{~Hz}, \mathrm{H}-1\right) ; 7.21-7.34(\mathrm{~m}, 5 \mathrm{H}, \mathrm{Ph}) ;{ }^{13} \mathrm{C}-\mathrm{NMR}\left(100 \mathrm{MHz}, \mathrm{CDCl}_{3}\right): \delta / \mathrm{ppm}=24.1$, 25.2, 25.4, $26.6\left(\mathrm{CH}_{3}, \mathrm{CH}_{3}(\mathrm{Cby})\right) ; 48.0\left(\mathrm{CH}_{2}, \mathrm{C}-2\right) ; 53.5\left(\mathrm{CH}_{2}, \mathrm{C}-3\right) ; 59.6,60.6\left(\mathrm{C}_{\mathrm{q}}, \mathrm{C}-4^{\prime}\right) ; 63.9$ $\left(\mathrm{CH}_{2}, \mathrm{C}-1\right)$; 76.1, $76.3\left(\mathrm{CH}_{2}, \mathrm{C}-3\right.$ ') ; 94.8, $95.8\left(\mathrm{C}_{\mathrm{q}}, \mathrm{C}-2\right.$ ') $)$ 126.9 (CH, C-7); 128.0, $128.3(2 \times \mathrm{CH}$, C-5, C-6); $140.1\left(\mathrm{C}_{\mathrm{q}}, \mathrm{C}-4\right) ; 151.9,152.6\left(\mathrm{C}_{\mathrm{q}}, \mathrm{C}-1^{\prime}\right)$; IR (Film): $\widetilde{v} / \mathrm{cm}^{-1}=3341$ (m, b, NH); 2979 , 2937, 2869, 2831 (s, C-H); 1697 (s, NC=O); 1455, 1410, 1377, 1368, 1337 (s, C-H); 1260, 1210, 1098, 1069 (s, C-O-C); 768, 737, 700 (s, Ph); MS (70 eV): m/z (\%)= $306(1)\left[\mathrm{M}^{+}\right], 133(100)$ $\left[(\mathrm{M}-\mathrm{OCby}-\mathrm{H})^{+}\right], 127$ (11) $\left[(\text { Cby-CO-H })^{+}\right], 120$ (22) $\left[\mathrm{BnNHCH}_{2}^{+}\right], 116$ (17), 91 (67) $\left[\mathrm{Bn}^{+}\right]$; calcd. For $\mathrm{C}_{17} \mathrm{H}_{26} \mathrm{~N}_{2} \mathrm{O}_{3}$ (306.40): C $66.64 \mathrm{H} 8.55$ N 9.14, found: C $66.35 \mathrm{H} 8.65$ N 9.05.

(1S)-2-(Benzylamino)-1-tributylstannylethyl

2,2,4,4-tetramethyl-1,3-oxazolidine-3carboxylate (7)

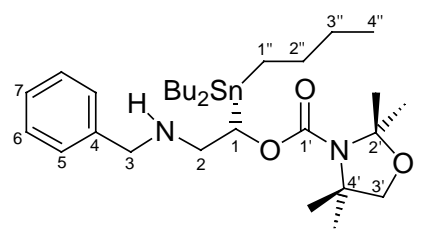

2-(Benzylamino)ethyl 2,2,4,4-tetramethyl-1,3-oxazolidine-3-carboxylate (4) (306 mg, $1.0 \mathrm{mmol}$ ) was dissolved in $3 \mathrm{~mL} \mathrm{CH}_{2} \mathrm{Cl}_{2}$ and the solution was cooled to $0{ }^{\circ} \mathrm{C}$. $\mathrm{NEt}_{3}(0.15 \mathrm{~mL}, 1.1 \mathrm{mmol}$, 1.1 equiv) and TBDMSOTf ( $0.25 \mathrm{~mL}, 1.1 \mathrm{mmol}, 1.1$ equiv) were added via syringe. The reaction mixture was stirred at room temperature for $30 \mathrm{~min}$ and subsequently $10 \mathrm{~mL}$ aq. $\mathrm{NaHCO}_{3}$ and 20 $\mathrm{mL} \mathrm{Et}_{2} \mathrm{O}$ were added. The organic layer was washed with $2 \times 5 \mathrm{~mL}$ aq. $\mathrm{NaHCO}_{3}, 5 \mathrm{~mL} \mathrm{H}_{2} \mathrm{O}$ and dried over anhydrous $\mathrm{Na}_{2} \mathrm{SO}_{4}$. The solvent was removed in vacuum to yield the TBDMSprotected Amine 5 as crude product. Subsequently $909 \mathrm{mg}(2.0 \mathrm{mmol})$ crude 2 - $(N$-benzyl- $N$-tertbutyldimethylsilyl-amino)ethyl 2,2,4,4-tetramethyl-1,3-oxazolidin-3-carboxylate (5) and $0.69 \mathrm{~mL}$ (3.0 mmol, 1.5 equiv) (-)-sparteine were dissolved in $8 \mathrm{~mL}$ THF and cooled to $-78{ }^{\circ} \mathrm{C}$. A solution of $s$-butyllithium in cyclohexane $(2.15 \mathrm{~mL}, 2.8 \mathrm{mmol}, 1.4$ equiv) was added within 5 min and the reaction mixture was stirred for $3.5 \mathrm{~h}$. $\mathrm{Bu}_{3} \mathrm{SnCl}(0.92 \mathrm{~mL}, 3.4 \mathrm{mmol}, 1.7$ equiv) was then added drop wise and the mixture was allowed to warm to room temperature over night. $10 \mathrm{~mL} 2 \mathrm{~N} \mathrm{HCl}$ were added and the mixture was stirred for $30 \mathrm{~min}$. After neutralization with $30 \mathrm{~mL}$ sat. $\mathrm{NaHCO}_{3}$-solution the mixture was extracted $\left(3 \times 20 \mathrm{~mL} \mathrm{Et}_{2} \mathrm{O}\right)$, washed with $5 \mathrm{~mL}$ sat. $\mathrm{NaHCO}_{3}$-solution and $5 \mathrm{~mL} \mathrm{H} \mathrm{H}_{2} \mathrm{O}$. The organic layer was dried over anhydrous $\mathrm{Na}_{2} \mathrm{SO}_{4}$ and the solvent was removed at the rotary evaporator. Flash chromatography $\left(\mathrm{Et}_{2} \mathrm{O} / \mathrm{PE}=1: 6 \rightarrow 1: 2\right)$ of the crude product gave $1.02 \mathrm{~g}(1.71 \mathrm{mmol}, 86 \%)$ stannane 7. 
An analogous reaction of $484 \mathrm{mg}(1.0 \mathrm{mmol})$ crude 2 - $(N$-benzyl- $N$-tert-butyldimethylsilylamino)-ethyl 2,2,4,4-tetramethyl-1,3-oxazolidine-3-carboxylate (5) with $0.23 \mathrm{~mL}$ (1.5 mmol, 1.5 equiv) TMEDA, $1.08 \mathrm{~mL}$ (1.4 mmol, 1.4 equiv) $s$-butyllithium (1.3 $\mathrm{M}$ in cyclohexane) and $0.46 \mathrm{~mL}$ (1.7 mmol, 1.7 equiv) $\mathrm{Bu}_{3} \mathrm{SnCl}$ in $4 \mathrm{~mL} \mathrm{Et}_{2} \mathrm{O}$ at $-78{ }^{\circ} \mathrm{C}$ gave $530 \mathrm{mg}(0.89 \mathrm{mmol}, 89 \%)$ rac-7.

${ }^{1} \mathrm{H}$ NMR $\left(400 \mathrm{MHz}, \mathrm{CDCl}_{3}\right): \delta / \mathrm{ppm}=0.88\left(\mathrm{t},{ }^{3} J_{3},{ }_{4}, "=7.3 \mathrm{~Hz}, 9 \mathrm{H}, \mathrm{H}-4{ }^{\prime \prime}\right)$ ); 0.81-0.98 (m, 6H, H1' '); 1.24-1.55 (m, 24H, H-2', H-3'', $\mathrm{CH}_{3}($ Cby)); 2.94-3.13 (m, 2H, H-2); 3.71 (s, 2H, H-3'); 3.76, $3.86\left(2 \times \mathrm{d},{ }^{2} J_{3 \mathrm{a}, 3 \mathrm{~b}}=13.1 \mathrm{~Hz}, 2 \mathrm{H}, \mathrm{H}-3\right)$; $4.92-4.98(\mathrm{~m}, 1 \mathrm{H}, \mathrm{H}-1)$; 7.21-7.26 (m, 1H, Ph); 7.28$7.31(\mathrm{~m}, 4 \mathrm{H}, \mathrm{Ph}) ;{ }^{13} \mathrm{C} \mathrm{NMR}\left(100 \mathrm{MHz}, \mathrm{CDCl}_{3}\right)$ : $\delta / \mathrm{ppm}=9.9\left(\mathrm{CH}_{2}, \mathrm{C}-1\right.$ '’ $) ; 13.7\left(\mathrm{CH}_{2}, \mathrm{C}-4\right.$ ' '); 24.1, 24.2, 25.2, 25.4, 26.5, $26.7\left(\mathrm{CH}_{3}, \mathrm{CH}_{3}(\mathrm{Cby})\right)$; $27.5\left(\mathrm{CH}_{2}, \mathrm{C}-2\right.$ '’ $) ; 29.1\left(\mathrm{CH}_{2}, \mathrm{C}-3\right.$ '’ $) ; 52.8$ $\left(\mathrm{CH}_{2}, \mathrm{C}-2\right)$; $53.4\left(\mathrm{CH}_{2}, \mathrm{C}-3\right)$; 59.4, $60.5\left(\mathrm{C}_{\mathrm{q}}, \mathrm{C}-4\right.$ ') $71.1(\mathrm{CH}, \mathrm{C}-1)$; 76.0, $76.2\left(\mathrm{CH}_{2}, \mathrm{C}-3\right.$ ' $)$; 94.5, $95.8\left(\mathrm{C}_{\mathrm{q}}, \mathrm{C}-2^{\prime}\right)$; $126.8(\mathrm{CH}, \mathrm{C}-7)$; 128.0, $128.3(2 \times \mathrm{CH}, \mathrm{C}-5, \mathrm{C}-6) ; 140.4\left(\mathrm{C}_{\mathrm{q}}, \mathrm{C}-4\right) ; 152.4,153.1$ $\left(\mathrm{C}_{\mathrm{q}}, \mathrm{C}-1\right.$ '); IR (film): $\tilde{v} / \mathrm{cm}^{-1}=2955,2924,2872,2851,2811$ (s, C-H); 1682 (s, NC=O); 1454, 1397, 1374 (s, C-H); 1260, 1093, 1063 (s, C-O-C); 769 (s, Ph); 698; MS (TOF-CI): m/z $(\%)=597(3)\left[(\mathrm{M}+\mathrm{H})^{+}\right], 539(100)\left[(\mathrm{M}-\mathrm{Bu})^{+}\right], 490(21)\left[(\mathrm{M}-\mathrm{Bn}-\mathrm{NH})^{+}\right], 291$ (20) $\left[\mathrm{SnBu}_{3}^{+}\right]$; calcd. For $\mathrm{C}_{29} \mathrm{H}_{52} \mathrm{~N}_{2} \mathrm{O}_{3} \mathrm{Sn}$ (595.46): C $58.50 \mathrm{H} 8.80 \mathrm{~N} 4.70$, found: C $58.22 \mathrm{H} 9.03 \mathrm{~N} \mathrm{4.53;}[\alpha]^{23}{ }_{D}$ $=+20.1\left(\mathrm{c}=0.76, \mathrm{CHCl}_{3}\right)$.

\section{[1S,2(2E)]-2-(N-Benzyl- $N$-(4-chlorobut-2-enyl)-amino)-1-tributylstannylethyl}

\section{tetramethyl-1,3-oxazolidine-3-carboxylate (8)}

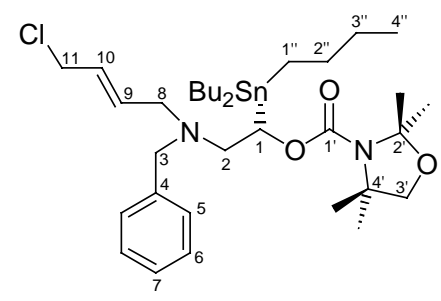

To a mixture of $595 \mathrm{mg}(1.0 \mathrm{mmol})$ (1S)-2-(benzylamino)-1-tributylstannylethyl 2,2,4,4tetramethyl-1,3-oxazolidine-3-carboxylate (7) and $530 \mathrm{mg}(5.0 \mathrm{mmol}, 5.0$ equiv) anhydrous $\mathrm{Na}_{2} \mathrm{CO}_{3}$ in $10 \mathrm{~mL} \mathrm{CH} \mathrm{CH}_{3} \mathrm{CN} 1.05 \mathrm{~mL}$ (10.0 mmol, 10 equiv) (E)-1,4-dichlorobut-2-ene were added and the reaction mixture was heated to reflux for $3 \mathrm{~h}$. After cooling down to room temperature and aqueous workup the product $8(604 \mathrm{mg}, 0.88 \mathrm{mmol}, 88 \%)$ was obtained by flash chromatography $\left(\mathrm{Et}_{2} \mathrm{O} / \mathrm{PE}=1: 19 \rightarrow 1: 4\right)$.

An analogous reaction of $456 \mathrm{mg}(0.77 \mathrm{mmol})$ rac-2-(benzylamino)-1-tributylstannylethyl 2,2,4,4-tetramethyl-1,3-oxazolidine-3-carboxylate (7) with $0.40 \mathrm{~mL}$ (3.83 mmol, 5.0 equiv) (E)1,4-dichlorobut-2-ene and $244 \mathrm{mg}$ (2.30 mmol, 3.0 equiv) anhydrous $\mathrm{Na}_{2} \mathrm{CO}_{3}$ in $5 \mathrm{~mL} \mathrm{CH}_{3} \mathrm{CN}$ gave $403 \mathrm{mg}(0.59 \mathrm{mmol}, 77 \%) \mathrm{rac}-\mathbf{8}$.

${ }^{1} \mathrm{H}$ NMR $\left(400 \mathrm{MHz}, \mathrm{CDCl}_{3}\right): \delta / \mathrm{ppm}=0.89\left(\mathrm{t},{ }^{3} J_{3},{ }_{4}, "=7.3 \mathrm{~Hz}, 9 \mathrm{H}, \mathrm{H}-4\right.$ '”); 0.79-0.96 (m, 6H, H1',); 1.24-1.62 (m, 24H, H-2', H-3', $\mathrm{CH}_{3}($ Cby)); 2.57-2.64 (m, 1H, H-2a); 2.70 (dt, $\left.{ }^{2} J_{8 \mathrm{a}, 8 \mathrm{~b}}=13.4 \mathrm{~Hz},{ }^{3} J_{8 \mathrm{a}, 9}=6.0 \mathrm{~Hz}, 1 \mathrm{H}, \mathrm{H}-8 \mathrm{a}\right) ; 3.08-3.16(\mathrm{~m}, 1 \mathrm{H}, \mathrm{H}-2 \mathrm{~b}) ; 3.20\left(\mathrm{dd},{ }^{2} J_{8 \mathrm{a}, 8 \mathrm{~b}}=13.4 \mathrm{~Hz}\right.$, $\left.1^{3} J_{8 \mathrm{~b}, 9}=6.0 \mathrm{~Hz}, \mathrm{H}, \mathrm{H}-8 \mathrm{~b}\right) ; 3.41\left(\mathrm{dd},{ }^{2} J_{3 \mathrm{a}, 3 \mathrm{~b}}=13.4 \mathrm{~Hz},{ }^{3} J_{3 \mathrm{a}, \mathrm{Ph}}=6.0 \mathrm{~Hz}, 1 \mathrm{H}, \mathrm{H}-3 \mathrm{a}\right) ; 3.73$ (s, 2H, H- 
3'); $3.79\left(\mathrm{~d},{ }^{2} J_{3 \mathrm{a}, 3 \mathrm{~b}}=13.4 \mathrm{~Hz}, 1 \mathrm{H}, \mathrm{H}-3 \mathrm{~b}\right) ; 4.02\left(\mathrm{~d},{ }^{3} \mathrm{~J}_{10,11}=6.3 \mathrm{~Hz}, 2 \mathrm{H}, \mathrm{H}-11\right) ; 5.02-5.14(\mathrm{~m}, 1 \mathrm{H}$, $\mathrm{H}-1)$; 5.72, $5.79\left(2 \times \mathrm{dt},{ }^{3} J_{9,10}=15.3 \mathrm{~Hz},{ }^{3} J_{10,11}=6.3 \mathrm{~Hz}, 2 \mathrm{H}, \mathrm{H}-9, \mathrm{H}-10\right) ; 7.19-7.23(\mathrm{~m}, 1 \mathrm{H}, \mathrm{Ph})$; 7.24-7.30 (m, 4H, Ph); ${ }^{13} \mathrm{C}$ NMR (100 MHz, $\left.\mathrm{CDCl}_{3}\right): \delta / \mathrm{ppm}=9.7\left(\mathrm{CH}_{2}, \mathrm{C}-1\right.$ '’); $13.7\left(\mathrm{CH}_{2}, \mathrm{C}-\right.$ 4' '); 24.2, 24.3, 25.4, 25.5, 26.5, $26.8\left(\mathrm{CH}_{3}, \mathrm{CH}_{3}(\mathrm{Cby})\right)$; $27.2\left(\mathrm{CH}_{2}, \mathrm{C}-2{ }^{\prime \prime}\right) ; 29.1\left(\mathrm{CH}_{2}, \mathrm{C}-3\right.$ '’ $)$; $44.7\left(\mathrm{CH}_{2}, \mathrm{C}-11\right)$; $54.2\left(\mathrm{CH}_{2}, \mathrm{C}-8\right)$; $57.4\left(\mathrm{CH}_{2}, \mathrm{C}-2\right)$; $58.0\left(\mathrm{CH}_{2}, \mathrm{C}-3\right) ; 59.5,60.5\left(\mathrm{C}_{\mathrm{q}}, \mathrm{C}-4\right.$ ' $)$; 67.5 (CH, C-1); 76.1, $76.4\left(\mathrm{CH}_{2}, \mathrm{C}-3\right.$ '); 94.8, 95.8 (C $\mathrm{q}, \mathrm{C}-2$ ') $) 126.9(\mathrm{CH}, \mathrm{C}-7)$; 128.1, $129.1(2 \times \mathrm{CH}$, C-5, C-6); 128.8 (CH, C-10); 132.5 (CH, C-9); $139.0\left(\mathrm{C}_{\mathrm{q}}, \mathrm{C}-4\right) ; 152.5,153.2\left(\mathrm{C}_{\mathrm{q}}, \mathrm{C}-1^{\prime}\right)$; IR (film): $\tilde{v} / \mathrm{cm}^{-1}=2956,2925,2872,2803$ (s, C-H); 1677 (s, NC=O); 1455, 1400, 1375 (s, C-H); 1258, 1094, 1063, 969 (s, C-O-C); 769, 741, 698; MS (TOF-CI): m/z (\%) = 685 (4) [(M+H) $\left.{ }^{+}\right]$, $649(12)\left[\left(\mathrm{M}-^{35} \mathrm{Cl}\right)^{+}\right], 627(100)\left[(\mathrm{M}-\mathrm{Bu})^{+}\right], 512(11), 490(91)\left[\left(\mathrm{M}-\mathrm{Bn}-\mathrm{N}-\mathrm{CH}_{2} \mathrm{CHCHCH}_{2} \mathrm{Cl}-\right.\right.$ $\mathrm{H})^{+}$], 468 (18), 291 (71) [ $\mathrm{SnBu}_{3}{ }^{+}$], 269 (24); calcd. For $\mathrm{C}_{33} \mathrm{H}_{57} \mathrm{ClN}_{2} \mathrm{O}_{3} \mathrm{Sn}$ (683.99): C $57.95 \mathrm{H}$ $8.40 \mathrm{~N}$ 4.10, found: C 58.16 H $8.64 \mathrm{~N} 3.89 ;[\alpha]^{23} \mathrm{D}=+21.2\left(\mathrm{c}=1.04, \mathrm{CHCl}_{3}\right)$.

(3R,4S)-3-(1-Benzyl-4-vinyl-pyrrolidin-3-yl)

2,2,4,4-tetramethyl-1,3-oxazolidin-3carboxylate (11)

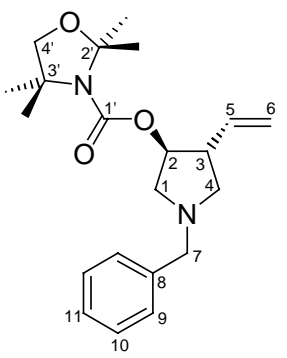

A solution of $114 \mathrm{mg}(0.16 \mathrm{mmol})$ stannane 8 in $2 \mathrm{~mL} \mathrm{Et} 2 \mathrm{O}$ was cooled to $-78{ }^{\circ} \mathrm{C}, 0.10 \mathrm{~mL}$ ( $0.25 \mathrm{mmol}, 1.5$ equiv) $n$-butyllithium $(2.5 \mathrm{M}$ in hexane) were added and the mixture was stirred at $-78{ }^{\circ} \mathrm{C}$ for $2 \mathrm{~h}$. For quenching the reaction $0.2 \mathrm{~mL} \mathrm{MeOH}$ and $0.1 \mathrm{~mL} \mathrm{H}_{2} \mathrm{O}$ were then added and the mixture was warmed to room temperature. The mixture was dried over anhydrous $\mathrm{Na}_{2} \mathrm{SO}_{4}$, the solvents were removed by rotary evaporation and the residue was purified by flash chromatography $\left(\mathrm{Et}_{2} \mathrm{O} / \mathrm{PE}=1: 20 \rightarrow 1: 4\right)$ to afford $57 \mathrm{mg}(0.16 \mathrm{mmol}, 95 \%)$ pyrrolidine 11.

${ }^{1} \mathrm{H}$ NMR (400 MHz, $\left.\mathrm{CDCl}_{3}\right): \delta / \mathrm{ppm}=1.39,1.40,1.53,1.55\left(4 \times \mathrm{s}, 12 \mathrm{H}, \mathrm{CH}_{3}(\right.$ Cby)); 2.28 (q, $\left.{ }^{3} J_{3,4 \mathrm{a}}={ }^{2} J_{4 \mathrm{a}, 4 \mathrm{~b}}=8.4 \mathrm{~Hz}, 1 \mathrm{H}, \mathrm{H}-4 \mathrm{a}\right) ; 2.77-2.83(\mathrm{~m}, 2 \mathrm{H}, \mathrm{H}-1)$; 2.85-2.93 (m, 1H, H-3); 3.01 (dd, $\left.{ }^{2} J_{4 \mathrm{a}, 4 \mathrm{~b}}=8.4 \mathrm{~Hz}, 1 \mathrm{H}, \mathrm{H}-4 \mathrm{~b}\right) ; 3.58,3.67\left(2 \times \mathrm{dd},{ }^{2} J_{7 \mathrm{a}, 7 \mathrm{~b}}=13.0 \mathrm{~Hz},{ }^{3} J_{7 \mathrm{a}, \mathrm{Ph}}=2.5 \mathrm{~Hz},{ }^{3} J_{7 \mathrm{~b}, \mathrm{Ph}}=3.1 \mathrm{~Hz}\right.$ 2H, H-7); $3.71,3.72$ (2×d, 2H, H-3'); 4.90-4.95 (m, 1H, H-2); 5.05, $5.11\left(2 \times \mathrm{d},{ }^{3} J_{5,6 \mathrm{Z}}=10.4 \mathrm{~Hz}\right.$, $\left.{ }^{3} J_{5,6 E}=17.1 \mathrm{~Hz}, 2 \mathrm{H}, \mathrm{H}-6\right) ; 5.84-5.93\left(\mathrm{~m},{ }^{3} J_{5,6 \mathrm{Z}}=10.4 \mathrm{~Hz},{ }^{3} J_{5,6 E}=17.1 \mathrm{~Hz}, 1 \mathrm{H}, \mathrm{H}-5\right) ; 7.21-7.35$ $(\mathrm{m}, 5 \mathrm{H}, \mathrm{Ph}) ;{ }^{13} \mathrm{C} \mathrm{NMR}\left(100 \mathrm{MHz}, \mathrm{CDCl}_{3}\right): \delta / \mathrm{ppm}=24.0,24.1,25.2,25.2,25.3,25.3,26.6,26.6$ $\left(\mathrm{CH}_{3}, \mathrm{CH}_{3}(\mathrm{Cby})\right)$; $48.8(\mathrm{CH}, \mathrm{C}-3)$; $58.0\left(\mathrm{CH}_{2}, \mathrm{C}-4\right)$; $59.6\left(\mathrm{CH}_{2}, \mathrm{C}-1\right) ; 59.8,60.5\left(\mathrm{C}_{\mathrm{q}}, \mathrm{C}-4\right.$ ') 59.9 $\left(\mathrm{CH}_{2}, \mathrm{C}-7\right)$; 76.0, $76.3\left(\mathrm{CH}_{2}, \mathrm{C}-3\right.$ '); $78.8(\mathrm{CH}, \mathrm{C}-2)$; 94.9, $95.7\left(\mathrm{C}_{\mathrm{q}}, \mathrm{C}-2\right.$ '); $115.7\left(\mathrm{CH}_{2}, \mathrm{C}-6\right)$; 126.9 (CH, C-11); 128.2, 128.5 (CH, C-9, C-10); 137.80, 137.83 (CH, C-5); 138.7 (C, C-8); 151.7, $152.4\left(\mathrm{C}_{\mathrm{q}}, \mathrm{C}-1^{\prime}\right)$; IR (film): $\tilde{v} / \mathrm{cm}^{-1}=3028,2980,2935,2867,2789$ (s, C-H); 1697 (s, $\mathrm{NC}=\mathrm{O})$; 1454, 1401, 1376, 1366, 1356, 1342, 1327 (s, C-H); 1260, 1209, 1152, 1098, 1069 (s, C-O-C); 914, 823, 769, 742, 701; MS (70 eV): m/z (\%) = $357(0.1)\left[(\mathrm{M}-\mathrm{H})^{+}\right], 343(0.3)[(\mathrm{M}-$ 
$\left.\left.\mathrm{CH}_{3}\right)^{+}\right], 185$ (100) $\left[(\mathrm{M}-\mathrm{HOCby})^{+}\right], 158$ (15) $\left[\left(\mathrm{M}-\mathrm{OCby}-\mathrm{C}_{2} \mathrm{H}_{4}, \mathrm{Cby}+2 \mathrm{H}\right)^{+}\right], 94$ (19), 91 (67) $\left[\mathrm{PhCH}_{2}{ }^{+}\right]$; calcd. For $\mathrm{C}_{21} \mathrm{H}_{30} \mathrm{~N}_{2} \mathrm{O}_{3}$ (358.48): C $70.36 \mathrm{H} 8.44 \mathrm{~N} 7.81$, found $\mathrm{C} 70.21 \mathrm{H} 8.65 \mathrm{~N}$ $7.65 ;[\alpha]^{23}=-39.0\left(\mathrm{c}=1.18, \mathrm{CHCl}_{3}\right)$.

(3S,4R)-1-Benzyl-4-vinylpyrrolidin-3-ol (14)

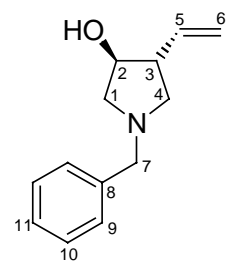

$75 \mathrm{mg}$ (0.21 mmol) pyrrolidine 11 were dissolved in $3 \mathrm{~mL} \mathrm{MeOH}$ and $0.07 \mathrm{~mL}$ (1.05 mmol, 5.0 equiv) methansulfonic acid were added. The mixture was heated under reflux for $3 \mathrm{~h} .578 \mathrm{mg}$ (4.18 mmol, 20 equiv) $\mathrm{K}_{2} \mathrm{CO}_{3}$ and $8 \mathrm{~mL} \mathrm{MeOH}$ were then added and the mixture was refluxed for additional $18 \mathrm{~h}$. After cooling to room temperature $10 \mathrm{~mL} \mathrm{H} \mathrm{H}_{2} \mathrm{O}$ and $10 \mathrm{~mL} \mathrm{CH}_{2} \mathrm{Cl}_{2}$ were added and the aqueous layer was extracted with $\mathrm{CH}_{2} \mathrm{Cl}_{2}(3 \times 5 \mathrm{~mL})$. The combined organic layers were dried $\left(\mathrm{Na}_{2} \mathrm{SO}_{4}\right)$, the solvents were removed in vacuum and the residue was purified by flash chromatography $(\mathrm{Cy} / \mathrm{EE}=8: 1 \rightarrow \mathrm{EE})$ to afford $33 \mathrm{mg}(0.12 \mathrm{mmol}, 56 \%)$ 3-hydroxy-pyrrolidine 14.

${ }^{1} \mathrm{H}$ NMR $\left(300 \mathrm{MHz}, \mathrm{CDCl}_{3}\right): \delta / \mathrm{ppm}=2.17\left(\mathrm{dd},{ }^{2} J_{4 \mathrm{a}, 4 \mathrm{~b}}=7.9 \mathrm{~Hz},{ }^{3} J_{3,4 \mathrm{a}}=9.4 \mathrm{~Hz}, 1 \mathrm{H}, \mathrm{H}-4 \mathrm{a}\right) ; 2.60$ $\left(\mathrm{dd},{ }^{2} J_{1 \mathrm{a}, 1 \mathrm{~b}}=10.1 \mathrm{~Hz},{ }^{3} J_{1 \mathrm{a}, 2}=5.9 \mathrm{~Hz}, 1 \mathrm{H}, \mathrm{H}-1 \mathrm{a}\right) ; 2.65-2.69\left(\mathrm{~m},{ }^{3} J_{3,4 \mathrm{a}}=9.4 \mathrm{~Hz},{ }^{3} J_{3,5}=8.0 \mathrm{~Hz}, 1 \mathrm{H}\right.$, H-3); $2.75\left(\mathrm{dd},{ }^{3} J_{1 \mathrm{~b}, 2}=3.0 \mathrm{~Hz},{ }^{2} J_{1 \mathrm{a}, 1 \mathrm{~b}}=10.1 \mathrm{~Hz}, 1 \mathrm{H}, \mathrm{H}-1 \mathrm{~b}\right) ; 3.05\left(\mathrm{dd},{ }^{2} J_{4 \mathrm{a}, 4 \mathrm{~b}}=7.9 \mathrm{~Hz}, 1 \mathrm{H}, \mathrm{H}-4 \mathrm{~b}\right)$; $3.60,3.61\left(2 \times \mathrm{s},{ }^{2} J_{7 \mathrm{a}, 7 \mathrm{~b}}=13.0 \mathrm{~Hz}, 2 \mathrm{H}, \mathrm{H}-7\right)$; 3.98-4.05 (m, 1H, H-2); 5.01, 5.07 (2×ddd, $\left.{ }^{3} J_{3,5}=8.0 \mathrm{~Hz}, \quad{ }^{3} J_{5,6 Z}=10.2 \mathrm{~Hz}, \quad{ }^{3} J_{5,6 E}=17.2 \mathrm{~Hz}, \quad 2 \mathrm{H}, \quad \mathrm{H}-6\right) ; \quad 5.79 \quad\left(\mathrm{ddd}, \quad{ }^{3} J_{3,5}=8.0 \mathrm{~Hz}\right.$, $\left.{ }^{3} J_{5,6 \mathrm{Z}}=10.2 \mathrm{~Hz},{ }^{3} J_{5,6 E}=17.2 \mathrm{~Hz} ; 1 \mathrm{H}, \mathrm{H}-5\right) ; 7.19-7.36(\mathrm{~m}, 5 \mathrm{H}, \mathrm{Ph}) ;{ }^{13} \mathrm{C} \mathrm{NMR}(75 \mathrm{MHz}$, $\left.\mathrm{CDCl}_{3}\right): \delta / \mathrm{ppm}=52.7(\mathrm{CH}, \mathrm{C}-3) ; 58.3\left(\mathrm{CH}_{2}, \mathrm{C}-4\right) ; 60.2\left(\mathrm{CH}_{2}, \mathrm{C}-1\right) ; 61.9\left(\mathrm{CH}_{2}, \mathrm{C}-7\right) ; 77.0(\mathrm{CH}$, C-2); $115.2\left(\mathrm{CH}_{2}, \mathrm{C}-6\right) ; 127.1$ (CH, C-11); 128.3, 128.8 (CH, C-9, C-10); 138.5 (CH, C-5); 138.7 $\left(\mathrm{C}_{\mathrm{q}}, \mathrm{C}-8\right)$; IR (film): $\widetilde{v} / \mathrm{cm}^{-1}=3372$ (s, b, OH); 3081, 3063, 3030 (s, CH=CH ); 2951, 2917, 2794 (s, C-H); 1640 (s, NC=O); 1496, 1474, 1455, 1376 (s, C-H); 1334 (s, OH); 1140, 1074, 1030 (s, C-O); 994, 916 (s, C=C); 756, 701; MS (70 eV): m/z (\%) = 203 (100) [ $\left.\mathrm{M}^{+}\right], 159$ (7), 133 (31), 91 (50) $\left[\mathrm{PhCH}_{2}^{+}\right]$; HRMS-ESI calcd. for $\mathrm{C}_{13} \mathrm{H}_{17} \mathrm{NO}$ (203.29): $[\mathrm{M}+\mathrm{H}]^{+}=204.1392$ found: $[\mathrm{M}+\mathrm{H}]^{+}=204.1383$. 
(3S,4R)-1-Benzyl-4-vinylpyrrolidin-3-yl (2R)-3,3,3-trifluoro-2-methoxy-2-phenylpropanoate (15)

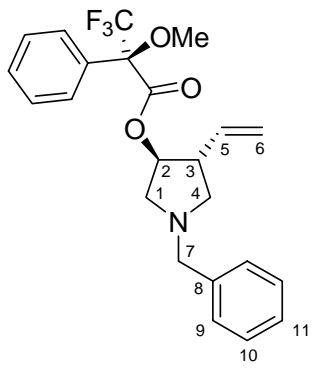

$10 \mathrm{mg}$ (0.05 mmol) 3-hydroxy-pyrrolidine 14 were solved in $5 \mathrm{~mL}$ pyridine and $0.25 \mathrm{~mL}$ (0.05 mmol) (R)-3,3,3-trifluoro-2-methoxy-2-phenylpropanoyl chloride $\left(0.2 \mathrm{M}\right.$ in $\left.\mathrm{CCl}_{4}\right)$ were added. The mixture was stirred at room temperature over night and was quenched with $2 \mathrm{~mL}$ water and $10 \mathrm{~mL}$ MTBE. The aqueous layer was extracted with MTBE $(3 \times 5 \mathrm{~mL})$, the combined organic layers were dried $\left(\mathrm{Na}_{2} \mathrm{SO}_{4}\right)$ and the solvents were removed under reduced pressure. Flash chromatography on silica (P/MTBE $\rightarrow 10: 1 \rightarrow 5: 1 \rightarrow 2: 1)$ yielded $10 \mathrm{mg}(0.02 \mathrm{mmol}, 47 \%)$ of ester 15 as colourless oil.

${ }^{1} \mathrm{H} \mathrm{NMR}\left(400 \mathrm{MHz}, \mathrm{CDCl}_{3}\right): \delta / \mathrm{ppm}=2.30\left(\mathrm{~m}, 1 \mathrm{H}, \mathrm{H}-1_{\mathrm{a}}\right) ; 2.81-2.89\left(\mathrm{~m},{ }^{2} J_{3 \mathrm{a}, 3 \mathrm{~b}}=8.6 \mathrm{~Hz}, 3 \mathrm{H}, \mathrm{H}-4\right.$, $\mathrm{H}-3) ; 2.99\left(\mathrm{~m}, 1 \mathrm{H}, \mathrm{H}-1_{\mathrm{b}}\right) ; 3.53\left(\mathrm{~s}, 3 \mathrm{H}, \mathrm{OCH}_{3}\right) ; 3.55\left(\mathrm{~d},{ }^{2} J_{7 \mathrm{a}, 7 \mathrm{~b}}=13.1 \mathrm{~Hz}, 1 \mathrm{H}, \mathrm{H}-7_{\mathrm{a}}\right) ; 3.67(\mathrm{~d}$, $\left.{ }^{2} J_{7 \mathrm{a}, 7 \mathrm{~b}}=13.1 \mathrm{~Hz}, 1 \mathrm{H}, \mathrm{H}-7_{\mathrm{b}}\right) ; 5.03-5.08\left(\mathrm{~m},{ }^{3} J_{6 \mathrm{E}, 5}=13.9 \mathrm{~Hz},{ }^{3} J_{6 \mathrm{Z}, 5}=4.2 \mathrm{~Hz}, 2 \mathrm{H}, \mathrm{H}-6\right) ; 5.20(\mathrm{~m}, 1 \mathrm{H}$, $\mathrm{H}-2)$; 5.79-5.89 (m, $\left.{ }^{3} J_{6 \mathrm{E}, 5}=13.9 \mathrm{~Hz},{ }^{3} J_{6 \mathrm{Z}, 5}=4.2 \mathrm{~Hz}, 1 \mathrm{H}, \mathrm{H}-5\right)$; 7.24-7.52 (m, 5H, Ph); ${ }^{9} \mathrm{~F}$ NMR $\left(282 \mathrm{MHz}, \mathrm{CDCl}_{3}\right): \delta / \mathrm{ppm}=-71.95 \quad\left(\mathrm{CF}_{3}\right) ;{ }^{9} \mathrm{~F} \quad \mathrm{NMR}$ (for rac-15) $\left(282 \mathrm{MHz}, \mathrm{CDCl}_{3}\right)$ : $\delta / \mathrm{ppm}=-71.89 \quad\left(\mathrm{CF}_{3}\right) ; \quad-71.96 \quad\left(\mathrm{CF}_{3}\right) ; \quad$ HRMS-ESI calcd. for $\mathrm{C}_{23} \mathrm{H}_{24} \mathrm{NO}_{3} \mathrm{~F}_{3}$ (419.17): $[\mathrm{M}+\mathrm{H}]^{+}=420.1787,[\mathrm{M}+\mathrm{Na}]^{+}=442.1606$, found: $[\mathrm{M}+\mathrm{H}]^{+}=420.1756,[\mathrm{M}+\mathrm{Na}]^{+}=442.1603$.

(1S)- $N$-Benzyl- $N$-(3-phenylprop-2-inyl)-1-tributylstannyl-2-aminoethyl 2,2,4,4-tetramethyl1,3-oxazolidine-3-carboxylate (9)

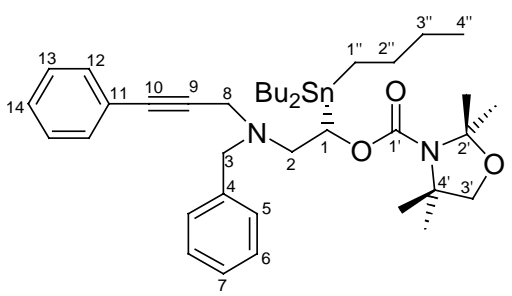

To a mixture of $357 \mathrm{mg}(0.60 \mathrm{mmol})$ (1S)-2-(benzylamino)-1-tributylstannylethyl-2,2,4,4tetramethyl-1,3-oxazolidin-3-carboxylate (7) and $318 \mathrm{mg}$ (3.0 mmol, 5.0 equiv) water free $\mathrm{Na}_{2} \mathrm{CO}_{3}$ in $5 \mathrm{~mL} \mathrm{CH}{ }_{3} \mathrm{CN}, 271 \mathrm{mg}$ (1.8 mmol, 3.0 equiv) 3-phenyl-2-propinyl chloride was added and the reaction mixture was heated to reflux for $3.5 \mathrm{~h}$. After cooling down to room temperature and aqueous workup the product 9 (404 $\mathrm{mg}, 0.57 \mathrm{mmol}$, 95\%) was obtained after flash chromatography $\left(\mathrm{Et}_{2} \mathrm{O} / \mathrm{PE}=1: 19 \rightarrow 1: 4\right)$.

${ }^{1} \mathrm{H}$ NMR $\left(300 \mathrm{MHz}, \mathrm{CDCl}_{3}\right): \delta / \mathrm{ppm}=0.86\left(\mathrm{t},{ }^{3} \mathrm{~J}_{3},{ }^{,}, ",=7.3 \mathrm{~Hz}, 9 \mathrm{H}, \mathrm{H}-4{ }^{\prime \prime}\right)$ ); 0.80-1.04 (m, 6H, H-

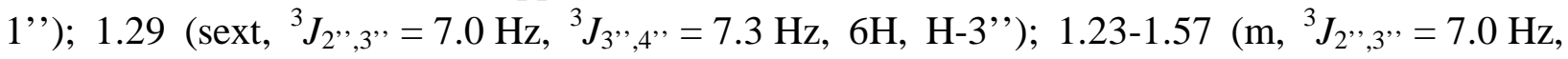


18H, H-2', $\mathrm{CH}_{3}(C b y)$ ); 2.94 (d, b, 1H, H-2a); 3.17 (dd, 1H, H-2b); 3.43 (d, ${ }^{2} J_{8 \mathrm{a}, 8 \mathrm{~b}}=17.6 \mathrm{~Hz}, 1 \mathrm{H}$, H-8a); 3.59-3.69 (m, $\left.{ }^{2} J_{3 \mathrm{a}, 3 \mathrm{~b}}=13.0 \mathrm{~Hz},{ }^{2} J_{8 \mathrm{a}, 8 \mathrm{~b}}=17.6 \mathrm{~Hz}, 2 \mathrm{H}, \mathrm{H}-3 \mathrm{a}, \mathrm{H}-8 \mathrm{~b}\right) ; 3.72$ (s, 2H, H-3'); $3.83\left(\mathrm{~d},{ }^{2} J_{3 \mathrm{a}, 3 \mathrm{~b}}=13.0 \mathrm{~Hz}, 1 \mathrm{H}, \mathrm{H}-3 \mathrm{~b}\right) ; 5.05-5.13(\mathrm{~m}, 1 \mathrm{H}, \mathrm{H}-1) ; 7.20-7.42(\mathrm{~m}, 10 \mathrm{H}, \mathrm{Ph}) ;{ }^{13} \mathrm{C}$ NMR $\left(75 \mathrm{MHz}, \mathrm{CDCl}_{3}\right): \delta / \mathrm{ppm}=9.8\left(\mathrm{CH}_{2}, \mathrm{C}-1{ }^{\prime}\right)$ ) $13.6\left(\mathrm{CH}_{2}, \mathrm{C}-4{ }^{\prime \prime}\right) ; 24.2,24.3,25.3,25.5,26.5,26.8$ $\left(\mathrm{CH}_{3}, \mathrm{CH}_{3}(\mathrm{Cby})\right) ; 27.1\left(\mathrm{CH}_{2}, \mathrm{C}-2{ }^{\prime \prime}\right) ; 29.1\left(\mathrm{CH}_{2}, \mathrm{C}-3\right.$ ') $) ; 41.2\left(\mathrm{CH}_{2}, \mathrm{C}-8\right) ; 57.3\left(\mathrm{CH}_{2}, \mathrm{C}-2\right) ; 58.2$ $\left(\mathrm{CH}_{2}, \mathrm{C}-3\right)$; 59.5, $60.5\left(\mathrm{C}_{\mathrm{q}}, \mathrm{C}-4\right.$ ') $) 67.7(\mathrm{CH}, \mathrm{C}-1)$; 76.2, $76.5\left(\mathrm{CH}_{2}, \mathrm{C}-3\right.$ ') ; 84.2, $85.9\left(\mathrm{CH}_{2}, \mathrm{C}-9\right.$, C-10); 94.8, 95.8 ( ( $\mathrm{q}, \mathrm{C}-2$ ') $123.5\left(\mathrm{C}_{\mathrm{q}}, \mathrm{C}-11\right)$; $127.0(\mathrm{CH}, \mathrm{C}-7)$; 127.9, 128.1 (2×CH, C-12, C$13)^{*} ; 128.2,129.2(2 \times \mathrm{CH}, \mathrm{C}-5, \mathrm{C}-6) ; 131.7(\mathrm{CH}, \mathrm{C}-14) * ; 138.8\left(\mathrm{C}_{\mathrm{q}}, \mathrm{C}-4\right) ; 152.6,153.3\left(\mathrm{C}_{\mathrm{q}}, \mathrm{C}-\right.$ 1'); IR (film): $\tilde{v} / \mathrm{cm}^{-1}=2960,2926,2870,2856$ (s, C-H); 1679 (s, NC=O); 1491, 1459, 1399, 1377, 1369, 1318 (s, C-H); 1262, 1206, 1095, 1065 (s, C-O-C); 772, 754 (s, C=C); 689; MS $(70 \mathrm{eV}): \mathrm{m} / \mathrm{z}(\%)=709(1)\left[(\mathrm{M}-\mathrm{H})^{+}\right], 653(81)\left[(\mathrm{M}-\mathrm{Bu})^{+}\right], 406(100)\left[(\text { CbyO-SnBu})^{+}\right], 335$ (19), 290 (19) [( $\left.\left.\mathrm{SnBu}_{3}-\mathrm{H}\right)^{+}\right], 246$ (50), 234 (20) $\left[\mathrm{SnBu}_{2}^{+}\right], 179$ (29), 177 (27) [SnBu $\left.{ }^{+}\right], 175$ (19), $156(31)\left[\mathrm{Cby}^{+}\right], 128(33)\left[(\mathrm{Cby}-\mathrm{CO})^{+}\right], 115(41), 98(45)\left[\mathrm{Cby}-\mathrm{OC}\left(\mathrm{CH}_{3}\right)_{2}{ }^{+}\right], 91(80)\left[\mathrm{Bn}^{+}\right], 59$ (92) [ $\mathrm{HOC}\left(\mathrm{CH}_{3}\right)_{2}{ }^{+}$], 55 (25); calcd. for $\mathrm{C}_{38} \mathrm{H}_{58} \mathrm{~N}_{2} \mathrm{O}_{3} \mathrm{Sn}$ (709.60): C 64.32 $\quad \mathrm{H} 8.24 \mathrm{~N} \quad 3.95$, found: C $64.70 \mathrm{H} 8.20 \mathrm{~N} \mathrm{3.80;}[\alpha]^{23}{ }_{\mathrm{D}}=+18.6\left(\mathrm{c}=0.96, \mathrm{CH}_{2} \mathrm{Cl}_{2}\right)$.

$(3 S, 4 E)$-1-Benzyl-4-benzylidenepyrrolidin-3-yl)

2,2,4,4-tetramethyl-1,3-oxazolidine-3carboxylate (13)

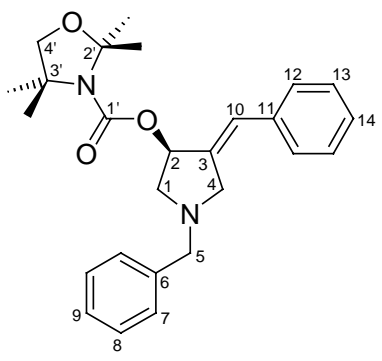

A solution of $106 \mathrm{mg}(0.15 \mathrm{mmol})$ stannane 9 in $2.5 \mathrm{~mL} \mathrm{Et}{ }_{2} \mathrm{O}$ was cooled to $-78{ }^{\circ} \mathrm{C}, 0.14 \mathrm{~mL}$ ( $0.225 \mathrm{mmol}, 1.5$ equiv) $n$-butyllithium $(2.5 \mathrm{M}$ in hexane) were added and the mixture was stirred at $-78^{\circ} \mathrm{C}$ for $2 \mathrm{~h}$. For quenching the reaction $0.2 \mathrm{~mL} \mathrm{MeOH}$ and $0.1 \mathrm{~mL} \mathrm{H}_{2} \mathrm{O}$ were then added and the mixture was warmed to room temperature. The mixture was dried over anhydrous $\mathrm{Na}_{2} \mathrm{SO}_{4}$, the solvents were removed by rotary evaporation and the residue was purified by flash chromatography $\left(\mathrm{PE} \rightarrow \mathrm{Et}_{2} \mathrm{O} / \mathrm{PE}=1: 3\right)$ to afford $50 \mathrm{mg}(0.12 \mathrm{mmol}, 79 \%)$ pyrrolidine 13.

${ }^{1} \mathrm{H}$ NMR $\left(300 \mathrm{MHz}, \mathrm{CDCl}_{3}\right): \delta / \mathrm{ppm}=1.39,1.40,1.43,1.46,1.56,1.57,1.60(7 \times \mathrm{s}, 12 \mathrm{H}$, $\mathrm{CH}_{3}($ Cby) $) ; 2.77\left(\mathrm{dd},{ }^{3} J_{4 \mathrm{a}, 2}=3.8 \mathrm{~Hz},{ }^{2} J_{4 \mathrm{a}, 4 \mathrm{~b}}=10.1 \mathrm{~Hz}, 1 \mathrm{H}, \mathrm{H}-4 \mathrm{a}\right) ; 2.90-2.98$ (m, 1H, H-1a); $3.40-$ 3.51 (m, 1H, H-1b); 3.73 (2×s, 4H, H-5, H-3'); 3.70-3.77 (m, $\left.{ }^{2} J_{4 \mathrm{a}, 4 \mathrm{~b}}=10.1 \mathrm{~Hz}, 1 \mathrm{H}, \mathrm{H}-4 \mathrm{~b}\right)$; 5.68$5.73(\mathrm{~m}, 1 \mathrm{H}, \mathrm{H}-2)$; 6.65-6.68 (m, 1H, H-10); 7.18-7.39 (m, 10H, 2×Ph); ${ }^{13} \mathrm{C} \mathrm{NMR} \mathrm{(75} \mathrm{MHz,}$ $\left.\mathrm{CDCl}_{3}\right): \delta / \mathrm{ppm}=24.2,25.3,25.6,26.5,26.8\left(\mathrm{CH}_{3}, \mathrm{CH}_{3}(\mathrm{Cby})\right) ; 56.8\left(\mathrm{CH}_{2}, \mathrm{C}-4\right) ; 59.6\left(\mathrm{CH}_{2}, \mathrm{C}-\right.$ 1); 60.0, $60.6\left(\mathrm{C}_{\mathrm{q}}, \mathrm{C}-4^{\prime}\right)$; $60.1\left(\mathrm{CH}_{2}, \mathrm{C}-5\right)$; $75.7(\mathrm{CH}, \mathrm{C}-2)$; 76.1, $76.4\left(\mathrm{CH}_{2}, \mathrm{C}-3\right.$ ') $78.8(\mathrm{CH}, \mathrm{C}-$ 2); 95.2, 95.8 (C $\mathrm{C}_{\mathrm{q}}, \mathrm{C}-2$ '); 126.3, 126.4, 127.1, 127.1, 128.3, 128.3, 128.4 (CH, C-7, C-8, C-9, C10, C-12, C-13, C-14); $137.0\left(\mathrm{C}_{\mathrm{q}}, \mathrm{C}-3\right)$; $138.7\left(\mathrm{C}_{\mathrm{q}}, \mathrm{C}-6\right)$; $139.7\left(\mathrm{C}_{\mathrm{q}}, \mathrm{C}-11\right)$; 151.9, $152.7\left(\mathrm{C}_{\mathrm{q}}, \mathrm{C}-\right.$ 1'); IR (film): $\tilde{v} / \mathrm{cm}^{-1}=3028,2981$ (m, C-H); 2936, 2873, 2791 (s, C-H); 1697 (s, NC=O); 
1495, 1453, 1397, 1379, 1349 (s, C-H); 1261, 1210, 1170, 1122, 1097, 1067 (s, C-O-C); 768, 755, 697; MS (70 eV): m/z (\%) = $247(100)\left[(\mathrm{M}-\mathrm{OCby}-\mathrm{H})^{+}\right], 156(31)\left[\mathrm{Cby}^{+}\right], 91(64)\left[\mathrm{Bn}^{+}\right]$; calcd. for $\mathrm{C}_{26} \mathrm{H}_{32} \mathrm{~N}_{2} \mathrm{O}_{3}$ (420.55): $\mathrm{C} 74.26 \mathrm{H} 7.67 \mathrm{~N} 6.66$, found: $\mathrm{C} 74.21 \mathrm{H} 7.56 \mathrm{~N} 6.51 ;[\alpha]^{23}{ }_{D}=$ $-25.2\left(\mathrm{c}=0.41, \mathrm{CH}_{2} \mathrm{Cl}_{2}\right)$. 